\title{
Direct benefit of vaccinating boys along with girls against oncogenic human papillomavirus: bayesian evidence synthesis
}

\author{
Johannes A Bogaards, ${ }^{12}$ Jacco Wallinga, ${ }^{2}$ Ruud H Brakenhoff, ${ }^{3}$ Chris J L M Meijer,, Johannes Berkhof
}

'Department of Epidemiology and Biostatistics, VU University Medical Centre, PO Box 7057, 1007 MB Amsterdam,

Netherlands

${ }^{2}$ Centre for Infectious Disease Control, National Institute for

Public Health and the

Environment, PO Box 1, 3720

BA Bilthoven, Netherlands

'Department of Otolaryngology/ Head and Neck Surgery, VU University Medical Centre, PO

Box 7057, 1007 MB Amsterdam, Netherlands

${ }^{4}$ Department of Pathology, VU University Medical Centre, PO Box 7057, 1007 MB Amsterdam, Netherlands

Correspondence to:

J A Bogaards

hans.bogaards@rivm.nl

Additional material is published online only. To view please visit

the journal online (http://dx.doi. org/10.1136/bmj.h2016)

Cite this as: BMJ 2015;350:h2016 doi: 10.1136/bmj.h2016

Accepted: 16 March 2015

\section{ABSTRACT}

OBJECTIVE

To assess the reduction in the vaccine preventable burden of cancer in men if boys are vaccinated along with girls against oncogenic human papillomavirus (HPV).

\section{DESIGN}

Bayesian evidence synthesis approach used to evaluate the impact of vaccination against HPV types 16 and 18 on the burden of anal, penile, and oropharyngeal carcinomas among heterosexual men and men who have sex with men. The reduced transmission of vaccine-type HPV from vaccination of girls was assumed to lower the risk of HPV associated cancer in all men but not to affect the excess risk of HPV associated cancers among men who have sex with men.

\section{SETTING}

General population in the Netherlands.

\section{INTERVENTION}

Inclusion of boys aged 12 into HPV vaccination programmes.

\section{MAIN OUTCOME MEASURES}

Quality adjusted life years (QALYs) and numbers needed to vaccinate.

\section{RESULTS \\ Before HPV vaccination, 14.9 (95\% credible interval 12.2 to 18.1) QALYs per thousand men were lost to vaccine preventable cancers associated with HPV in the Netherlands. This burden would be reduced by $37 \%$ (28\% to $48 \%)$ if the vaccine uptake among girls remains at the current level of $60 \%$. To prevent one additional case of cancer among men, 795 boys ( 660 to 987 ) would need to be vaccinated; with tumour}

\section{WHAT IS ALREADY KNOWN ON THIS TOPIC}

HPV is aetiologically linked to anogenital and oropharyngeal cancer in men and in women

HPV vaccine is offered free of charge to preadolescent girls; heterosexual men could indirectly benefit from a reduced transmission of vaccine type HPV

\section{WHAT THIS STUDY ADDS}

HPV vaccination of girls will not substantially influence the burden of anal cancer, disproportionately present in men who have sex with men, but is estimated to have a strong effect on the burden of oropharyngeal cancer

The added effect of vaccinating boys on the future occurrence of oropharyngeal cancer is considerable if $60 \%$ of the girls are vaccinated but is estimated to become small if uptake in girls reaches $90 \%$

At the current vaccine uptake level of $60 \%$ in girls in the Netherlands, the number of boys who would need to be vaccinated to prevent one cancer in men will be about four times as high as the number of girls needed to prevent one cervical cancer, indicating that a cost effective implementation of sex neutral vaccination requires low vaccine pricing specific numbers for anal, penile, and oropharyngeal cancer of 2162, 3486, and 1975, respectively. The burden of HPV related cancer in men would be reduced by $66 \%$ (53\% to 805$)$ if vaccine uptake among girls increases to $90 \%$. In that case, 1735 boys (1240 to 2900) would need to be vaccinated to prevent an additional case; with tumour specific numbers for anal, penile, and oropharyngeal cancer of 2593, 29107, and 6484 , respectively.

\section{CONCLUSIONS}

Men will benefit indirectly from vaccination of girls but remain at risk of cancers associated with HPV. The incremental benefit of vaccinating boys when vaccine uptake among girls is high is driven by the prevention of anal carcinomas, which underscores the relevance of HPV prevention efforts for men who have sex with men.

\section{Introduction}

Vaccination against human papillomavirus (HPV) has been implemented in many countries with the specific aim of protecting women against cervical cancer. ${ }^{12}$ Inclusion of boys into vaccination programmes for girls is expected to provide some benefit to women, although the benefit will be small if programmes already achieve high coverage among girls. ${ }^{3-11}$ Protection of women should, however, not be the sole public health objective of HPV vaccination because men are also at risk of vaccine preventable cancers. ${ }^{1213}$ Accumulation of evidence for the role of HPV in the aetiology of cancer in non-cervical sites, ${ }^{14-16}$ and demonstration that HPV vaccine is also effective in preventing non-cervical lesions in men as well as women, ${ }^{17} 18$ have prompted initiatives to vaccinate not only girls but also boys against HPV. ${ }^{1920}$ Currently, Australia is the only country with a publicly funded HPV immunisation campaign for men..$^{21}$

An often cited obstacle to male HPV vaccination is the prohibitive cost. ${ }^{22-24}$ An economic assessment based on market introduction prices concluded that inclusion of boys in the immunisation programme in the United States exceeded conventional thresholds of cost effective interventions. ${ }^{5}$ Vaccine prices, however, have declined since market introduction, and cost requirements need not deter universal vaccination today, even though HPV vaccines are still expensive relative to other vaccines. To decide whether boys should be offered HPV vaccination, we need to assess the burden of HPV associated disease in men and how much of this will be prevented by vaccination of girls. Unless vaccination of girls will eliminate vaccine-type HPV infection from the population, men will remain vulnerable to vaccine preventable cancers, especially men who have sex with men, who are disproportionately affected by HPV related cancer. ${ }^{25}$ 
The burden of HPV associated cancer in men derives primarily from neoplasms in the anogenital and head and neck regions. ${ }^{26}$ The connection between an anogenital or oral HPV infection and history of sexual behaviour is well established in epidemiological studies. ${ }^{27-31} \mathrm{In}$ particular, men who have sex with men are, compared with heterosexual men, at high risk for oral and anal HPV infections, ${ }^{3233}$ though not for penile HPV infections. ${ }^{3435}$ Hence, men who have sex with men are at increased risk particularly for anal cancer, especially when they also have HIV. ${ }^{25} 36-38$

Most anal cancers are thought to be caused by oncogenic HPV types. ${ }^{16}$ Head and neck neoplasms make up most HPV associated cancers in non-cervical sites, ${ }^{39}$ but there is no consensus about the attributable risk of HPV. Head and neck cancer consists of a wide variety of tumour entities, and the HPV positivity rate of anatomical subsites varies substantially. 4041 An aetiologic link with HPV has been convincingly demonstrated-based on prominent biological and epidemiological differences between HPV positive and HPV negative tumours-only for squamous cell carcinomas that arise in the oropharyngeal region. ${ }^{15}$ Even when restricted to oropharyngeal squamous cell carcinomas, reported HPV positivity rates vary considerably throughout the world because of differences in methods for HPV detection as well as varying exposure to HPV and competing risk factors, such as tobacco smoking and alcohol consumption.

We present a bayesian evidence synthesis of the incremental benefit of vaccinating boys along with girls in preventing HPV associated cancers in men. Our analysis concerned only the vaccination of preadolescent boys and did not consider targeted vaccination of men who have sex with men. We did, however, take full account of the disproportionally high burden of HPV related cancers in men who have sex with men, who are not protected by herd immunity from female vaccination. Previous analyses of the incremental benefit of vaccination of boys did not account for the concentrated burden of HPV related disease among men who have sex with men ${ }^{3-11}$ and could have overvalued the indirect protection from vaccination of girls. We explicitly considered the cancer burden among men who have sex with men by estimating population attributable fractions for the relevant tumour entities. Our analysis combined multiple sources of data to derive accurate estimates of the burden of HPV related cancer among men conditional on vaccination of girls in the Netherlands and to account for uncertainty in key parameters that govern the incremental benefit of vaccinating boys against HPV.

\section{Methods}

Cancer data

We based our analysis on recent data from the Dutch national cancer registry. ${ }^{4243}$ We considered only those malignancies with "strong" evidence for a causal link with HPV according to the International Agency for Research on Cancer (IARC). Currently, this includes cancers of the penis (ICD-10 (international classification of diseases, 10th revision) code C60), anus and anal canal (C21) and squamous cell carcinoma of the oropharynx, including base of the tongue and tonsils (C01, C09, C10). Over the period 2000-10, each year an average of 450 men were diagnosed with either of these cancers, comprising 114 penile cases, 61 anal cases, and 275 oropharyngeal cases a year. The European standardised rate (ESR) was 1.32 per 100000 men for penile cancer, 0.69 per 100000 men for anal cancer, and 3.10 per 100000 men for oropharyngeal cancer. The average ages at diagnosis over the period 2000-10 were 68, 62, and 60, respectively.

\section{Computational overview}

We measured the incremental effect of vaccinating boys against oncogenic HPV types 16 and 18 by quality adjusted life years (QALYs) gained in men and number of boys needed to vaccinate to prevent one cancer case. Epidemiological formulas for these quantities are presented in appendix 1 and are functions of age specific incidences of cancer, HPV aetiological fractions, and survival rates of people with HPV positive cancer.

We considered inclusion of boys aged 12 into the current HPV immunisation programme in the Netherlands, which started in 2009 with a catch-up campaign targeting girls up until age 17 before routine vaccination of girls aged 12 . We did not consider catch-up vaccination for boys. Incremental effects of vaccinating boys aged 12 are presented without incorporation of herd immunity-that is, before introduction of HPV vaccine in the Netherlands; conditional on vaccination of girls aged 12 with an uptake of $60 \%$, which was the uptake in 2012 among girls aged 12 in the Netherlands; and conditional on vaccination of girls with an uptake of $90 \%$, the target uptake for vaccines in the Dutch national immunisation programme. ${ }^{44}$ The reduction in HPV infection rates in men from vaccination of girls was estimated from a heterosexual transmission model, fitted to cross sectional and longitudinal data of high risk HPV infections before the introduction of HPV vaccine in the Netherlands. ${ }^{4546}$ The transmission model predicts the reduction in risk of infection with HPV 16 or 18 among heterosexual men, given a particular vaccine uptake among girls aged 12. The reduction in risk of heterosexual infection with HPV 16 and 18 from vaccination of girls was subsequently projected onto the burden of HPV associated cancers in men that are attributable to heterosexual transmission-that is, the complement of the fraction attributable to homosexuality in men.

We estimated attribution of male homosexuality to the burden of HPV related cancer by site specific population attributable fractions. A population attributable fraction is a comparison of incidence under the observed pattern of exposure with the incidence under a counterfactual pattern in which exposure is entirely absent from the population. ${ }^{47}$ Here, the observed pattern of exposure is a male population with a proportion men who have sex with men, the counterfactual pattern is a completely heterosexual male population, and the population attributable fraction combines the 
prevalence of men who have sex with men in the adult male population with the relative risk for HPV associated cancers among men who have sex with men versus heterosexual males. ${ }^{364}$ Men who have sex with men are thus expected to enjoy the same absolute reduction in the risk of HPV associated cancers as heterosexual men, but the reduced transmission of HPV 16 and 18 from vaccination of girls will not affect the excess risk of HPV associated cancers among men who have sex with men (fig 1).

\section{Model parameters}

We adopted a bayesian evidence synthesis approach in which we combined multiple sources of evidence and accounted for uncertainty in key parameters. Data on cancer incidence and survival were obtained from the Dutch national cancer registry, and data sources for HPV attributable fractions were chosen to reflect unbiased estimates with regard to the Netherlands. We obtained parameters from meta-analyses when possible, but we limited our estimates to those studies that used a validated method of HPV DNA detection. The HPV attributable risk of anal cancer was obtained from international studies ${ }^{16}$ because the fraction of HPV positive anal tumours displays little variation across studies. In the case of penile and oropharyngeal cancer, we obtained HPV attributable risks from local tumour samples ${ }^{49-51}$ because the estimates of HPV prevalence showed considerable heterogeneity across studies in meta-analyses. ${ }^{5253}$ With regard to oropharyngeal cancer, this variation is partly due to methodological issues and partly due to spatiotemporal variation in the attributable fraction of HPV in the oropharynx. To avoid use of inflated estimates of the fraction of oropharyngeal squamous cell carcinomas that can be prevented by HPV vaccination, we restricted our estimate of HPV attributable risk to recently obtained biopsies on which high quality histological auditioning was performed using a validated test algorithm for the detection of HPV in head and neck tumour samples. ${ }^{54}$ We obtained estimates of distribution of HPV type in HPV positive cancers from published systematic reviews as studies displayed little variation with regard to the relative contribution of types 16 and 18 among the HPV positive cases. ${ }^{415253}$ Survival rates in penile and oropharyngeal cancers accounted for the relatively favourable prognosis of HPV positive carcinomas. ${ }^{49} 55$ Prior distributions for the various parameters related to disease burden are described in appendix 2 and are summarised as median values with $95 \%$ credible intervals in table 1.

We used cancer specific valuations for quality of life (-that is, utilities) that would apply to most patients for the period starting after the primary treatment effects have resolved, which is of significant frequency and duration to be useful for modelling preventive interventions. ${ }^{56}$ The prophylactic efficacy of vaccinating boys aged 12 against HPV 16/18 specific cancers in men was set equal to the efficacy of vaccinating preadolescent girls against HPV 16/18 positive cervical intraepithelial neoplasia grade 2 or worse (CIN2+).

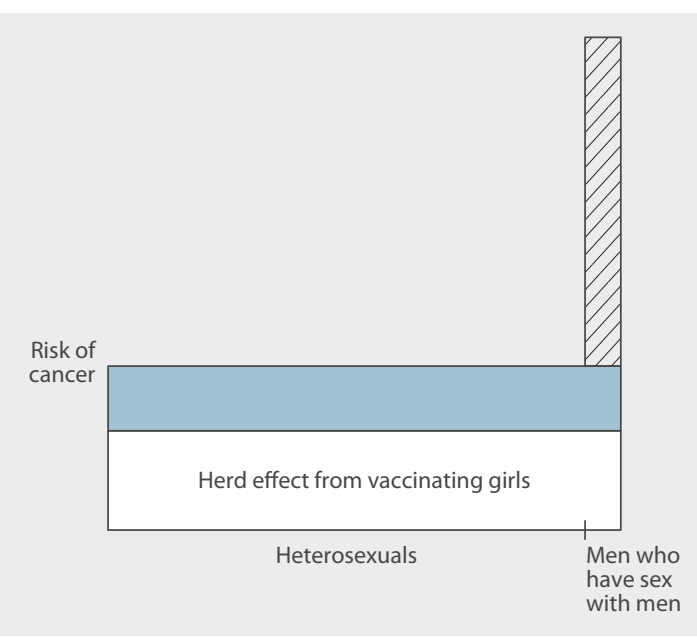

Fig 1 Clustering of HPV related tumours in men who have sex with men. Number of men who have sex with men in population is represented by bar width. Herd immunity from vaccination of girls (area of white rectangle) is assumed not to affect number of cases of cancer attributable to male homosexuality (hatched area). Population attributable fraction is hatched area relative to total area

Table 1 | Epidemiologic parameters for estimating burden of HPV related cancer in men in the Netherlands

\begin{tabular}{|c|c|}
\hline $\begin{array}{l}\text { Cancer site (ICD code) and summary } \\
\text { measure }\end{array}$ & $\begin{array}{l}\text { Median value }(95 \% \\
\text { credible interval)* }\end{array}$ \\
\hline \multicolumn{2}{|l|}{ Penis (ICD-C60) } \\
\hline Lifetime risk of diagnosis & $172(160$ to 186$) \times 10^{-5}$ \\
\hline HPV attributable fraction & $36 \%(45 \% \text { to } 65 \%)^{50}$ \\
\hline Proportion HPV 16 positivet & $60 \%(56 \% \text { to } 64 \%)^{52}$ \\
\hline Proportion HPV 18 positivet & $13 \%(11 \% \text { to } 16 \%)^{52}$ \\
\hline 10 year relative survival probability $\ddagger$ & 0.65 (0.58 to 0.71$)$ \\
\hline HPV positive hazard ratio & $0.21(0.06 \text { to } 0.76)^{49}$ \\
\hline \multicolumn{2}{|l|}{ Anus and anal canal (ICD-C21) } \\
\hline Lifetime risk of diagnosis & $74(68$ to 80$) \times 10^{-5}$ \\
\hline Relative risk among MSM§ & $31(8.2 \text { to } 80)^{36}$ \\
\hline HPV attributable fraction & $86 \%(83 \% \text { to } 89 \%)^{16}$ \\
\hline Male:female odds ration & $0.29(0.19 \text { to } 0.44)^{16}$ \\
\hline Proportion HPV 16 positive & $85 \%(82 \% \text { to } 87 \%)^{53}$ \\
\hline Proportion HPV 18 positivet & $7 \%(5 \% \text { to } 10 \%)^{53}$ \\
\hline 10 year relative survival probability $\ddagger$ & 0.45 (0.38 to 0.52$)$ \\
\hline \multicolumn{2}{|l|}{ Oropharynx (ICD-C01, C09, C10) } \\
\hline Lifetime risk of diagnosis & 305 (295 to 316$) \times 10^{-5}$ \\
\hline Relative risk among MSM§ & $2.9(1.3 \text { to } 5.4)^{36}$ \\
\hline HPV attributable fraction & $29 \%(19 \% \text { to } 41 \%)^{51}$ \\
\hline Male:female odds ration & $3.5(1.4 \text { to } 8.6)^{51}$ \\
\hline Proportion HPV 16 positivet & $87 \%(83 \% \text { to } 90 \%)^{41}$ \\
\hline Proportion HPV 18 positive & $3 \%(1 \% \text { to } 5 \%)^{41}$ \\
\hline 10 year relative survival probability $\ddagger$ & $0.23(0.20$ to 0.25$)$ \\
\hline HPV positive hazard ratio & $0.46(0.37 \text { to } 0.57)^{55}$ \\
\hline \multicolumn{2}{|c|}{$\begin{array}{l}\text { *Data from Dutch national cancer registry unless reference number shown. } \\
\text { +Relative contribution among HPV positive cases. } \\
\text { fCancer specific survival among men aged } 45-74 \text {; younger men have } \\
\text { better survival, older men have worse survival (data not shown). } \\
\text { \$Relative risk of cancer among men who have sex with men (MSM) } v \\
\text { heterosexual men. } \\
\text { १Odds ratio for HPV positivity of cancer cases among men compared } \\
\text { with women. }\end{array}$} \\
\hline
\end{tabular}

The meta-analytic estimate of the latter quantity, obtained by combining per protocol efficacy estimates from vaccine trials in women, ${ }^{5758}$ was 0.98 (95\% credible interval 0.95 to 0.99 ). 


\section{Sensitivity analysis}

In our base case analyses, we assumed that the degree of clustering of HPV related tumours in men who have sex with men is determined by the prevalence of such men in the population and their relative risks of HPV associated cancers irrespective of aetiology. We increased the degree of clustering by assuming that all HPV positive anal carcinomas are attributable to male homosexuality, effectively assuming that heterosexual men have zero risk for HPV related anal cancer. Secondly, we assumed that the excess risk for oropharyngeal cancer in men who have sex with men is entirely due to HPV positive squamous cell carcinomas (fig 1). These alternative scenarios lead to a stronger incremental effect of vaccinating boys on the burden of HPV associated cancers in men.

In base case analyses, we considered only the direct benefit to the boys vaccinated, which does not depend on vaccine uptake in other men. Numbers needed to vaccinate will depend on male vaccine coverage if one also considers the extra herd immunity in non-vaccinated men that results from vaccinating boys in addition to girls. In appendix 1 we accounted for additional herd immunity in non-vaccinated heterosexual men and recalculated the number of boys aged 12 who would need to be vaccinated to prevent one cancer or gain one QALY. The projected vaccine coverage of boys was set at $30 \%, 50 \%$, and $70 \%$, provided vaccine coverage in girls was $60 \%$ and $90 \%$. Note that the total benefit of male vaccination is still underestimated because our model does not allow for quantification of herd immunity in non-vaccinated men who have sex with men resulting from a reduced homosexual transmission.

\section{Results}

Burden of HPV associated cancers in men before vaccination

We estimate that 16.8 (95\% credible interval 13.7 to 20.3) QALYs per thousand men were lost to HPV related cancers before HPV vaccination in the Netherlands. Vaccine preventabletypes-that is, HPV 16 and 18-accounted for $89 \%$ ( $86 \%$ to $92 \%$ ) of the total burden of HPV related cancer among men. The projected loss due to vaccine preventable cancers was 14.9 (12.2 to 18.1) QALYs per thousand men, of which 8.4 (6.0 to 11.3) QALYs per thousand men were lost to oropharyngeal squamous cell carcinomas, 5.2 (4.5 to 6.0) to anal carcinomas, and 1.1 (0.7 to 2.4 ) to penile cancer. Despite the high proportion of HPV 16/18 positive cases, anal carcinomas contributed only $35 \%$ ( $28 \%$ to $43 \%)$ to the vaccine preventable burden of cancer before vaccination.

Indirect effect of vaccinating girls on HPV associated cancers in men

Vaccination of girls reduces the HPV prevalence throughout the heterosexual population, and the lifetime risk of HPV 16 and 18 infection for heterosexual men declines with increasing vaccine uptake among girls. The predicted decline is smaller for HPV 16 than for HPV 18 because of the higher transmission potential of HPV 16 (fig 2). At 60\% vaccine uptake among girls, the estimated reduction in lifetime risk of infection for heterosexual men is 53\% (95\% credible interval $43 \%$ to $62 \%$ ) for HPV 16 and $62 \%$ (56\% to 68\%) for HPV 18. The burden of vaccine preventable cancer reduces by $37 \%$ ( $28 \%$ to $48 \%$ ). Projected reductions in the burden of cancer in men vary across tumour sites (fig 3), with reductions of 54\% (46\% to 62\%) for penile cancer and 46\% (35\% to 57\%) for oropharyngeal cancer; but the burden of anal cancer decreases by only $18 \%$ (6\% to $37 \%)$

At $90 \%$ vaccine uptake among girls, reductions in risk of infection are $94 \%$ (95\% credible interval $90 \%$ to 98\%) for HPV 16 and 97\% (93\% to 100\%) for HPV 18, and the estimated reduction in the HPV 16/18 specific cancer burden among men is $66 \%$ (53\% to $80 \%)$. The burden of penile cancer decreases by $95 \%$ ( $91 \%$ to $98 \%$ ) and the burden of oropharyngeal cancer decreases by $84 \%$ ( $67 \%$ to $94 \%)$. The burden of anal cancer decreases by only $32 \%$ (11\% to $65 \%$ ), and anal carcinomas constitute $73 \%$ (52\% to $88 \%$ ) of the remaining burden of vaccine preventable cancer among men.

\section{Incremental benefit of vaccinating boys on HPV associated cancers in men}

If girls had not been vaccinated against oncogenic HPV, then vaccinating boys at the age of 12 would have led to a gain of 14.6 (95\% credible interval 11.9 to 17.7 ) QALYs per 1000 boys vaccinated (table 2). This corresponds to 69 (56 to 84) boys who would need to be vaccinated to gain one QALY, 78 (63 to 96) boys who would need to be vaccinated to gain one life year, and 466 (405 to 542)
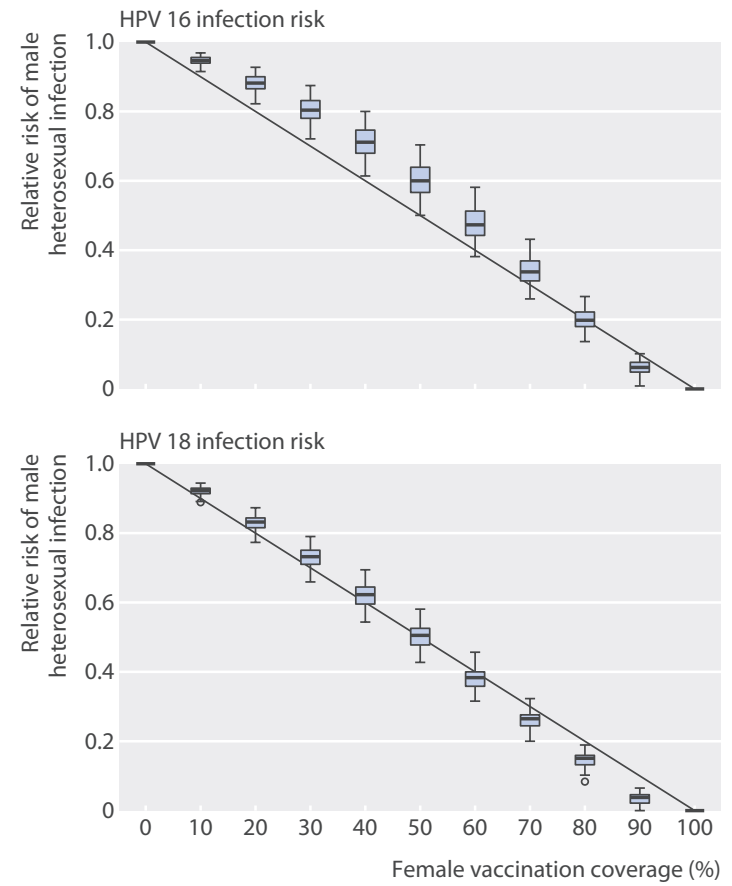

Fig 2 | Lifetime risk of infection with HPV 16 or HPV 18 among heterosexual men relative to scenario without vaccination as function of vaccine coverage of girls. Box plots show variation from uncertainty about type specific parameters in HPV transmission model fitted to HPV infections in the Netherlands 


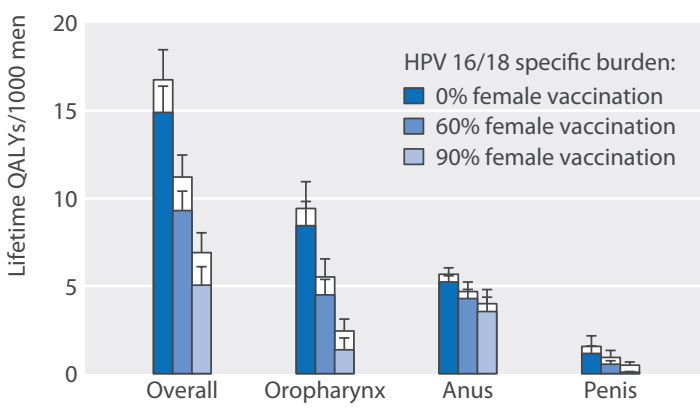

Fig 3 | Burden of HPV associated cancers in men in the Netherlands in relation to vaccine coverage of girls, overall and separately for carcinomas of oropharynx, anus, and penis. White bars (posterior median plus 1SD) denote QALYs lost to all HPV types, whereas coloured bars denote estimated burden from vaccine preventable HPV types 16 and 18

boys who would need to be vaccinated to prevent one case of cancer (table 3).

If girls aged 12 continue to be vaccinated at the current uptake of $60 \%$ in the Netherlands, then the incremental gain from vaccinating boys aged 12 is 9.1 ( $95 \%$ credible interval 7.1 to 11.4) QALYs per 1000 vaccines boys vaccinated, corresponding to 109 (88 to 140) boys who would need to be vaccinated to gain one QALY. The number of boys who would need to be vaccinated to prevent one case of cancer is 795 (660 to 987). Tumour specific numbers needed to prevent one case would be 1975 (1405 to 2849) for oropharyngeal cancer, 2162 (1810 to 2869) for anal cancer, and 3486 (2710 to 4650) for penile cancer.

If vaccine uptake among girls aged 12 reaches the target of $90 \%$, the incremental gain from vaccinating boys aged 12 will become 4.8 (95\% credible interval 2.8 to 6.9) QALYs per 1000 boys vaccinated. This gain can

Table 2 | Potential benefit of vaccinating boys aged 12 against HPV 16/18 in the Netherlands*. Figures are quality adjusted life years (QALYs) to be gained per 1000 boys vaccinated ( $95 \%$ credible interval)

\begin{tabular}{llll} 
& \multicolumn{4}{l}{ Vaccine coverage among girlst } \\
\cline { 2 - 4 } Cancer site (ICD-10 code) & $\mathbf{0 \%}$ & $\mathbf{6 0 \%}$ & $\mathbf{9 0 \%}$ \\
\hline Penis (C60) & $1.1(0.7$ to 2.3$)$ & $0.5(0.3$ to 1.1$)$ & $0.1(0.0$ to 0.2$)$ \\
\hline Anus and anal canal (C21) & $5.1(4.4$ to 5.9$)$ & $4.2(3.1$ to 5.1$)$ & $3.5(1.8$ to 4.8$)$ \\
\hline Oropharynx (C01, C09, C10) & $8.3(5.9$ to 11.1$)$ & $4.4(2.9$ to 6.3$)$ & $1.3(0.5$ to 2.9$)$ \\
\hline Any cancer & $14.6(11.9$ to 17.7$)$ & $9.1(7.1$ to 11.4$)$ & $4.8(2.8$ to 6.9$)$ \\
\hline
\end{tabular}

*Assuming similar efficacy against cancers as against high grade cervical intraepithelial neoplasia. tThree dose uptake among girls aged $12: 60 \%$ is current level and $90 \%$ is target level.

Table 3 | Numbers of boys who would need to be vaccinated ( $95 \%$ credible interval) to prevent outcomes related to HPV $16 / 18$ infection in men*

\begin{tabular}{llll} 
& \multicolumn{2}{l}{ Vaccine coverage among girlst } \\
\cline { 2 - 4 } Outcome prevented & $\mathbf{0 \%}$ & $\mathbf{6 0 \%}$ & $\mathbf{9 0 \%}$ \\
\hline Penile cancer & $1595(1314$ to 2010$)$ & $3486(2710$ to 4650$)$ & $29107(16828$ to 79557$)$ \\
\hline Anal cancer & $1769(1605$ to 1954$)$ & $2162(1810$ to 2869$)$ & $2593(1934$ to 5129$)$ \\
\hline Oropharyngeal cancer & $1048(803$ to 1441$)$ & $1975(1405$ to 2849$)$ & $6484(3037$ to 16534$)$ \\
\hline Any cancer & $466(405$ to 542$)$ & $795(660$ to 987$)$ & $1735(1240$ to 2900$)$ \\
\hline Life year lost & $78(63$ to 96$)$ & $124(98$ to 159$)$ & $231(161$ to 393$)$ \\
\hline QALY lost & $69(56$ to 84$)$ & $109(88$ to 140$)$ & $203(143$ to 347$)$ \\
\hline
\end{tabular}

QALY=quality adjusted life year.

*Assuming similar efficacy against cancers as against high grade cervical intraepithelial neoplasia.

tThree dose uptake among girls aged 12: 60\% is current level and $90 \%$ is target level. be largely attributed to the prevention of anal carcinomas, responsible for 3.5 (95\% credible interval 1.8 to 4.8) QALYs per 1000 boys vaccinated. An estimated 203 (143 to 347) boys would need to be vaccinated to gain one QALY and 1735 (1240 to 2900) to prevent one cancer case. The predominance of anal cancer in the incremental benefit of vaccinating boys becomes apparent in tumour specific numbers needed to vaccinate. At 90\% vaccine uptake among girls, 2593 (1934 to 5129) boys need to be vaccinated to prevent one case of anal cancer, 6484 (3037 to 16534) to prevent one case of oropharyngeal cancer, and 29107 (16828 to 79557) to prevent one case of penile cancer.

\section{Sensitivity analysis}

Attribution of all HPV positive anal carcinomas to men who have sex with men increases the effect of vaccination of boys from 9.1 to 10.1 ( $95 \%$ credible interval 8.4 to 12.2) QALYs per 1000 boys vaccinated at $60 \%$ uptake among girls. The effect of vaccination further increases to 11.0 (8.9 to 13.6) QALYs if we also assume that the excess risk for oropharyngeal cancer in men who have sex with men is entirely due to HPV positive squamous cell carcinomas. At 90\% vaccine uptake among girls, the effect of vaccinating boys increases from 4.8 to 6.5 (5.4 to 8.2) QALYs per 1000 boys vaccinated if all HPV positive anal carcinomas are attributed to men who have sex with men. The gain further increases to 8.0 (5.8 to 11.9) QALYs if we also assume a stronger clustering of HPV positive oropharyngeal cancer among men who have sex with men versus heterosexual men.

The number of boys vaccinated per cancer prevented at $60 \%$ vaccine coverage in girls decreased from 795 among boys vaccinated to 639 (95\% credible interval 527 to 788 ) if extra herd immunity from heterosexual transmission reduction was included at 50\% vaccine coverage among boys (table 4). The number of boys vaccinated per QALY gained likewise decreased from 109 to 91 (72 to 115). At 90\% female vaccine coverage, these figures decreased from 1735 to 1483 (1085 to 2388) and from 203 to 181 (130 to 295), respectively, in a cohort of men with $50 \%$ vaccinated. The numbers of boys vaccinated per cancer case prevented or per QALY gained were even lower if we considered $30 \%$ instead of $50 \%$ vaccine coverage among boys. Additional herd immunity in men has less impact on the number vaccinated per cancer prevented if uptake among boys is higher because the scope for indirect effects gets smaller the more boys are vaccinated.

\section{Discussion}

We have presented a detailed analysis of the burden of HPV associated cancers in men in the Netherlands and of the incremental benefit of including boys into HPV vaccination programmes conditional on vaccine coverage in girls. We estimated that about 15 QALYs per 1000 men were lost to vaccine preventable cancers before the introduction of HPV vaccine, and that the current HPV vaccination programme targeting only girls reduces this burden by $37 \%$ if the current vaccine uptake of $60 \%$ among preadolescent girls is sustained. 


\begin{tabular}{|c|c|c|c|}
\hline \multirow{2}{*}{$\begin{array}{l}\text { Outcome } \\
\text { prevented }\end{array}$} & \multicolumn{3}{|c|}{ Projected vaccine coverage in boys } \\
\hline & $30 \%$ & $50 \%$ & $70 \%$ \\
\hline \multicolumn{4}{|c|}{ At $60 \%$ vaccine coverage in girlst } \\
\hline Any cancer & 609 (495 to 761) & 639 (527 to 788) & $676(563$ to 827$)$ \\
\hline QALY lost & $86(68$ to 110$)$ & $91(72$ to 115$)$ & 94 (76 to 119$)$ \\
\hline \multicolumn{4}{|c|}{ At $90 \%$ vaccine coverage in girlst } \\
\hline Any cancer & 1401 (1016 to 2223) & 1483 (1085 to 2388) & 1611 (1160 to 2602) \\
\hline QALY lost & 176 (127 to 281$)$ & 181 (130 to 295) & 192 (137 to 314) \\
\hline
\end{tabular}

If boys are included in the vaccination programme, about 110 boys would need to be vaccinated to gain one QALY and around 800 to prevent one case of cancer. These figures are helpful for prioritising male HPV vaccination and provide indispensable input for health economic evaluations.

\section{Strengths and limitations of this study}

Our approach was deliberately conservative in the sense that we avoided inflated estimates of the vaccine preventable burden of cancer in men. Firstly, we did not include cancer of the oral cavity in our analysis because the causal association with HPV is still unclear. ${ }^{59}$ Also the incidence of such cancer in the Netherlands is about as high as the incidence of anal, penile, and oropharyngeal cancers combined. ${ }^{42}$ Nonetheless, the estimated burden of HPV associated cancers in men will be only marginally affected by inclusion of cancer of the oral cavity when the HPV attributable risk is about $5 \% .^{6061}$ Secondly, we did not extrapolate the upward trend in HPV related oropharyngeal cancer that has been reported for various countries ${ }^{516263}$ into the future. By including only biopsies collected during 2008-11, our estimate of the HPV attributable risk for oropharyngeal squamous cell carcinomas was already substantially higher than the average over the period 1990-2010.51 Extrapolation from past trends into the future could result in a biased assessment as it is not clear what caused the increase in HPV positivity rates over previous decades. Thirdly, in the base case we conservatively assumed that the increased risks of oropharyngeal and anal carcinomas for men who have sex with men apply equally to HPV positive and HPV negative tumours. A stronger clustering of HPV positive carcinomas among men who have sex with men led to substantially greater estimates of the benefit that men could derive from HPV vaccination and a weaker association between the incremental benefit of vaccinating boys and vaccine coverage in girls. The potential benefit of vaccinating boys would be improved under these assumptions, but it remains to be determined whether heterosexual men have zero risk for HPV positive anal cancer and to what extent men who have sex with men are at increased risk for HPV positive oropharyngeal squamous cell carcinomas.

Our analysis depends on a previously published type specific HPV transmission model to account for herd effects from vaccination of girls. The herd effect only appears as an input parameter in calculations as the computational framework is further composed of classic epidemiological formulas. The parameters in our analysis (other than those describing herd effects) were taken directly from the literature, with inclusion of statistical uncertainty. An advantage from this approach is that it circumvents the need to calibrate a complex model for the transmission of multiple HPV types and natural history of various carcinomas to infection and disease endpoints. A limitation is that we lose some of the detail that can be built into a complex simulation model such as, for instance, clustering of vaccine uptake in sexual couples.

Our approach also circumvents the need for developing an explicit model for site specific HPV transmission among heterosexuals and men who have sex with men, which greatly reduces model complexity and avoids explication of numerous uncertain parameters, such as transmission probabilities for site specific modes of transmission. This is critical as relatively little is known about the natural course of non-cervical cancers. In particular, we assumed that heterosexual men are indirectly protected against vaccine preventable disease through a reduced exposure to HPV 16/18 infection in women, but we did not specify a site specific degree of protection. The underlying argument is that we considered sexual partnerships as the basis for HPV transmission and infection of specific anatomical sites as secondary. ${ }^{66}$ It is conceivable, however, that the risk of oropharyngeal HPV infection is mediated by different behavioural characteristics than those captured in our transmission model, which could lead to different reductions in risk of infection for oropharyngeal compared with anogenital cancers. For example, a reduced transmissibility of HPV during oral sex relative to anogenital intercourse would lead to stronger risk reductions for oropharyngeal cancer than for anal or penile cancer. We also assumed that the efficacy of HPV vaccination in reducing female to male transmission rates will be similar to the efficacy that has been shown against CIN2+ in women. Efficacy against transient HPV 16 infection, however, defined as any time DNA detection or six month persistent infection, is in the order of $80 \% .{ }^{667}$ If transient infections in vaccinated women still contribute to secondary transmission, our estimates of reduction in risk of infection might be too optimistic.

There have been a few earlier accounts in modelling studies of the increased risk of HPV related cancers among men who have sex with men. Burger and colleagues systematically reduced the herd immunity benefits to all men from vaccination of girls to assess the impact of men who have sex with men on the incremental cost effectiveness of male vaccination in sensitivity analyses, ${ }^{64}$ whereas Smith and colleagues previously assumed that anal cancers occur exclusively in men who have sex with men. ${ }^{10}$ To our knowledge, Laprise and colleagues are the only ones to have previously considered the prevalence of men who have sex with men and their disease specific relative risks versus male heterosexuals in a modelling study. ${ }^{65}$ They explicitly 
incorporated sexual orientation in the model contact structure to deal with clustering of HPV related disease in men who have sex with men and assumed that such men are not protected by vaccination of girls whatsoever. In our approach, vaccination of girls will lower the risk of HPV associated cancer among all men but will not affect the excess risk of HPV associated cancers in men who have sex with men. Our approach thus acknowledges the possibility that some of the herd immunity benefits from vaccination of girls might propagate to the population of men who have sex with men through bisexual behaviour, although the reductions in risk of infection for strictly homosexual men might be slightly overestimated.

We set the prophylactic efficacy of HPV vaccination against HPV 16/18 related cancers in men equal to the figure reported for HPV 16/18 positive CIN2+ in women, which has a meta-analytic estimate close to $100 \%{ }^{5758}$ This choice can be criticised as the efficacy of HPV vaccines in preventing oropharyngeal infections or lesions has not yet been shown. ${ }^{20}$ The efficacy of the bivalent vaccine against prevalent oral HPV 16/18 infections four years after vaccination has been estimated at 0.93 (95\% confidence interval 0.63 to 1.00 ), which is not significantly different from protection against persistent cervical infections. ${ }^{68}$ The efficacy of the quadrivalent HPV vaccine against vaccine type anogenital lesions in male vaccine trials has been estimated at 0.90 (0.69 to 0.98), although efficacy against anal intraepithelial neoplasia associated with vaccine types was only 0.78 (0.40 to 0.93) in per protocol analyses. ${ }^{1718}$ The relatively low efficacy against anal compared with cervical intraepithelial neoplasia might be explained by the fact that male study participants had a longer sexual history than participants in HPV vaccine trials among young women. ${ }^{5758}$

Our study pinpoints which parameters govern the incremental benefit of male HPV vaccination. At moderate uptake among girls, the key parameters are related to reductions in heterosexual transmission from vaccination of girls and the aetiologic fraction of HPV in oropharyngeal cancer. If vaccine uptake among girls approaches $90 \%$, the key parameters will be predominantly related to men who have sex with men, who are not protected by reduced heterosexual transmission. As of now, anal carcinomas contribute less to the burden of vaccine preventable cancers in men than might be expected on the basis of their large HPV attributable risk, but they will constitute the major burden of HPV related cancer if vaccine uptake among preadolescent girls continues to rise. ${ }^{44}$

\section{Implications and considerations for HPV vaccination campaigns}

The causal relation of HPV with cervical cancer was recognised before HPV was implied in the aetiology of cancer in non-cervical sites. The decision to offer vaccination freely to preadolescent girls was motivated by considerations of burden of disease and cost effectiveness. The burden of cervical cancer before HPV vaccination had been estimated at 50-60 QALYs per 1000 women, ${ }^{69}$ up to four times the burden of anal, penile, and oropharyngeal cancer in men combined. In the Netherlands the number of girls that needed to be vaccinated to gain one QALY from cervical disease prevention was about 25 and around 200 to prevent one case of cervical cancer. At the current $60 \%$ vaccine uptake among preadolescent girls in the Netherlands, 109 boys would need to be vaccinated to gain one male QALY and around 800 to prevent one case of cancer among men. These numbers are substantially higher than those that motivated vaccination of girls to protect women against cervical cancer, even if we consider all tumours that have an acknowledged link with HPV. Provision of HPV vaccine to women thus constitutes equitable policy if one adheres to the view that those most affected by disease should be considered first in a rational resource allocation scheme. The burden of HPV related cancer among men is nonetheless substantial, even after herd immunity from vaccination of girls is taken into account.

Our estimate of 15 vaccine preventable QALYs lost per 1000 men translates into a total loss of 1500 QALYs per 100000 boys, the approximate male birth cohort size in the Netherlands. For comparison, the burden of acute infection with hepatitis B virus in the Netherlands has recently been estimated at around 1300 disability adjusted life years. ${ }^{70}$ This figure also includes those carriers of hepatitis B that cannot be prevented by childhood vaccination in the Netherlands-for example, from immigration from high endemic countries. It follows that HPV vaccination of boys aged 12 compares favourably with universal infant vaccination against hepatitis B, which has been added to the Dutch national immunisation programme in 2011.

The efficiency of vaccinating boys aged 12 against oncogenic HPV needs ultimate assessment in a health economic evaluation where discounted future benefits are compared with discounted net costs of extending the vaccination programme to men. Costs of vaccine purchase and delivery precede the health benefits by about half a century. Hence, at an annual discount rate of $3 \%$, the number of boys who would need to be vaccinated to gain one discounted QALY will be about four to six times that in the setting without discounting. This implies that if the net costs exceed $€ 100$ (£72; \$107) per vaccinated boy, sex neutral vaccination is unlikely to be economically feasible. Note, however, that tender pricing and the recent switch to a two dose vaccination schedule in the Netherlands might already have created a situation where sex neutral vaccination is a cost effective strategy.

Sex neutral vaccination of preadolescents might ultimately lead to control of HPV related diseases in men and women alike but will not protect currently sexually active men who have sex with men, who are facing an increasing risk of HPV related cancer. They could substantially benefit from targeted prevention strategies, including vaccination, screening for anal intraepithelial neoplasia, or a combination of both. The effect of targeted prevention of men who have sex with men will not markedly depend on vaccine uptake among girls, and age specific strategies need to be evaluated in 
conjunction with universal preadolescent vaccination. Whether targeted prevention of men who have sex with men will be effective depends on several factors, including HPV and HIV co-infection status and awareness in the target group for participation in disease prevention programmes. As a comparison, in Amsterdam by 2011 the targeted hepatitis B vaccination programme, which has been operational in the Netherlands since 1998, reached an estimated $30-38 \%$ of men who have sex with men. ${ }^{71}$ This vaccine coverage was high enough to cause a marked decrease in the incidence of acute hepatitis B, probably because those who engage most in high risk sex were reached. ${ }^{71}$ It should be noted, however, that HPV is transmitted more easily than hepatitis B. Previous exposure to HPV might influence vaccine efficacy, which has been shown only in men with a limited number of lifetime sexual partners. ${ }^{1718}$ Recommendations for targeted prevention strategies should take account of specific transmission routes among men who have sex with men, of the natural history of anal cancer, and of the modulatory effects of HIV infection. ${ }^{72}$

\section{Conclusions}

In summary, we have presented a comprehensive analysis of the incremental benefit of vaccinating boys along with girls against oncogenic HPV infection. Authorities should first and foremost strive to vaccinate as many girls as possible. A vaccine coverage of $90 \%$ in girls, however, might not be realistic for many countries, including the Netherlands, where uptake among preadolescent girls was fairly constant between $50 \%$ and $60 \%$ over the past five years. Inclusion of boys into preadolescent HPV vaccination programmes is warranted once the incremental costs of vaccination conform to society's willingness to pay in comparison with the incremental health effects. This balance will depend on vaccine price and on the coverage that is already achieved among girls. While our analysis shows the size of the indirect benefits that accrue from vaccination of girls, our estimates underscore the continued relevance of HPV prevention efforts for men, specifically those who are disproportionally affected by HPV related disease. The predominance of anal cancer in the burden of HPV related disease at high vaccine coverage of girls emphasises the need for a better understanding of the epidemiology of HPV infection among men who have sex with men in countries where a satisfactory vaccine uptake among preadolescent girls can be attained. Further research should delineate ways to promote prevention of HPV induced diseases in populations that are hard to reach or derive little benefit from a reduced transmission in the general population, including but not limited to men who have sex with men. In any case, protection of women should no longer be the sole public health objective of any HPV vaccination programme.

Contributors: JAB and JB conceived and designed the analysis. JAB performed the analysis and wrote the manuscript and supplementary annex. JB was closely involved in the writing process. JW, RHB, and CJLM contributed to interpretation of data and critical revision of the manuscript. $J A B$ is guarantor.
Funding: This research was supported by the EU Seventh Framework Programme of DG Research through the PREHDICT and COHEAHR projects, which received co-funding by the Health Research and Development Council of the Netherlands Organization for Scientific Research (ZonMW 121030032). The funders had no role in study design, data collection and analysis, decision to publish, or preparation of the manuscript.

Competing interests: All authors have completed the ICMJE uniform disclosure form at www.icmje.org/coi_disclosure.pdf and declare:

$\mathrm{JB}$ has received fees for participation in expert meetings organised by GSK and Merck, CM has received fees for participation in expert meetings organised by Merck, outside the submitted work. The other authors have no potential conflicts of interest.

Ethical approval: Not required.

Transparency: The lead author (the manuscript's guarantor) affirms that this manuscript is an honest, accurate, and transparent account of the study being reported; that no important aspects of the study have been omitted; and that any discrepancies from the study as planned (and, if relevant, registered) have been explained.

Data sharing: Technical appendix, statistical code, and datasets are available from the corresponding author.

This is an Open Access article distributed in accordance with the Creative Commons Attribution Non Commercial (CC BY-NC 4.0) license, which permits others to distribute, remix, adapt, build upon this work non-commercially, and license their derivative works on different terms, provided the original work is properly cited and the use is non-commercial. See: http://creativecommons.org/licenses/ by-nc/4.0/.

Franceschi S, Denny L, Irwin KL, et al. Eurogin 2010 roadmap on cervical cancer prevention. Int J Cancer 2011;128:2765-74

2 Li N, Franceschi S, Howell-Jones R, et al. Human papillomavirus type distribution in 30,848 invasive cervical cancers worldwide: variation by geographical region, histological type and year of publication. Int J Cancer 2011;128:927-35.

3 Taira AV, Neukermans CP, Sanders GD. Evaluating human papillomavirus vaccination programs. Emerg Infect Dis 2004;10:1915-23.

4 Jit M, Choi YH, Edmunds WJ. Economic evaluation of human papillomavirus vaccination in the United Kingdom. BMJ 2008;337:a769.

5 Kim JJ, Goldie SJ. Cost effectiveness analysis of including boys in a human papillomavirus vaccination programme in the United States. BM/ 2009:339:b3884.

6 Elbasha EH, Dasbach EJ. Impact of vaccinating boys and men against HPV in the United States. Vaccine 2010;28:6858-67.

Bogaards JA, Kretzschmar M, Xiridou M, et al. Sex-specific immunization for sexually transmitted infections such as human papillomavirus: insights from mathematical models. PLoS Med 2011;8:e1001147.

8 Brisson $\mathrm{M}$, van de Velde N, Franco EL, et al. Incremental impact of adding boys to current human papillomavirus vaccination programs: role of herd immunity. J Infect Dis 2011;204:372-6.

9 Chesson HW, Ekwueme DU, Saraiya M, et al. The cost-effectiveness of male HPV vaccination in the United States. Vaccine 2011:29:8443-50.

10 Smith MA, Lew JB, Walker RJ, et al. The predicted impact of HPV vaccination on male infections and male HPV-related cancers in Australia. Vaccine 2011:29:9112-22.

11 Marty R, Roze S, Bresse X, et al. Estimating the clinical benefits of vaccinating boys and girls against HPV-related diseases in Europe. BMC Cancer 2013:13:10.

12 Low GM, Attiga YS, Garg G, et al. Can male vaccination reduce the burden of human papillomavirus-related disease in the United States? Viral Immunol 2012;25:174-86

13 Stanley M. Vaccinate boys too. Nature 2012;488(suppl 7413):S10.

14 Gillison ML, Koch WM, Capone RB, et al. Evidence for a causal association between human papillomavirus and a subset of head and neck cancers. J Natl Cancer Inst 2000;92:709-20.

15 D'Souza G, Kreimer AR, Viscidi R, et al. Case-control study of human papillomavirus and oropharyngeal cancer. N Engl J Med 2007:356:1944-56.

16 De Vuyst H, Clifford GM, Nascimento MC, et al. Prevalence and type distribution of human papillomavirus in carcinoma and intraepithelial neoplasia of the vulva, vagina and anus: a meta-analysis. Int J Cancer 2009:124:1626-36

17 Giuliano AR, Palefsky JM, Goldstone S, et al. Efficacy of quadrivalent HPV vaccine against HPV infection and disease in males. N Engl J Med 2011;364:401-11.

18 Palefsky JM, Giuliano AR, Goldstone S, et al. HPV vaccine against anal HPV infection and anal intraepithelial neoplasia. N Engl/ Med 2011;365:1576-85

19 Centers for Disease Control and Prevention (CDC). Recommendations on the use of quadrivalent human papillomavirus vaccine in 
males-Advisory Committee on Immunization Practices (ACIP), 2011. MMWR Morb Mortal Wkly Rep 2011;60:1705-8.

20 Georgousakis M, Jayasinghe S, Brotherton J, et al. Population-wide vaccination against human papillomavirus in adolescent boys: Australia as a case study. Lancet Infect Dis 2012;12:627-34.

21 Kirby T. Australia to be first country to vaccinate boys against HPV. Lancet Oncol 2012;13:e333.

22 Castle PE, Scarinci I. Should HPV vaccine be given to men? BMJ 2009;339:b4127.

23 Cuschieri K. Should boys receive the human papillomavirus vaccine? No. BMJ 2009;339:b4921

24 Hibbitts S. Should boys receive the human papillomavirus vaccine? Yes. BM/ 2009;339:b4928.

25 Lawton MD, Nathan M, Asboe D. HPV vaccination to prevent anal cancer in men who have sex with men. Sex Transm Infect 2013;89:342-343.

26 Forman D, de Martel C, Lacey CJ, et al. Global burden of human papillomavirus and related diseases. Vaccine 2012;30(suppl 5):F12-23.

27 Kreimer AR, Alberg AJ, Daniel R, et al. Oral human papillomavirus infection in adults is associated with sexual behavior and HIV serostatus. J Infect Dis 2004;189:686-98.

28 Chin-Hong PV, VittinghoffE, Cranston RD, et al. Age-specific prevalence of anal human papillomavirus infection in HIV-negative sexually active men who have sex with men: the EXPLORE study. J Infect Dis 2004;190:2070-6

29 D'Souza G, Agrawal Y, Halpern I, et al. Oral sexual behaviors associated with prevalent oral human papillomavirus infection. J Infect Dis 2009;199:1263-9.

30 Nyitray AG, Carvalho da Silva RJ, et al. Age-specific prevalence of and risk factors for anal human papillomavirus (HPV) among men who have sex with women and men who have sex with men: the HPV in men (HIM) study. J Infect Dis 2011;203:49-57.

31 Heiligenberg M, Alberts CJ, Waterboer T, et al. Route of sexual exposure is independently associated with seropositivity to HPV-16 and HPV-18 among clients of an STI clinic in the Netherlands. J Infect Dis 2013;208:1081-5.

32 Del Amo J, González C, Geskus RB, et al. What drives the number of high-risk human papillomavirus types in the anal canal in HIV-positive men who have sex with men? J Infect Dis 2013;207:1235-41.

33 Mooij SH, Boot HJ, Speksnijder AG, et al. Oral human papillomavirus infection in HIV-negative and HIV-infected MSM. AIDS 2013;27:2117-28.

34 Van Aar F, Mooii SH, van der Sande MA, et al. Anal and penile high-risk human papillomavirus prevalence in HIV-negative and HIV-infected MSM. AIDS 2013;27:2921-31.

35 Vriend HJ, Bogaards JA, van der Klis FR, et al. Patterns of human papillomavirus DNA and antibody positivity in young males and females, suggesting a site-specific natural course of infection. PLOS One 2013; e60696.

36 Frisch M, Smith E, Grulich A, et al. Cancer in a population-based cohort of men and women in registered homosexual partnerships. Am J Epidemiol 2003;157:966-72.

37 Hessol NA, Pipkin S, Schwarcz S, et al. The impact of highly active antiretroviral therapy on non-AIDS-defining cancers among adults with AIDS. Am J Epidemiol 2007;165:1143-53.

38 Patel P, Hanson DL, Sullivan PS, et al. Incidence of types of cancer among HIV-infected persons compared with the general population in the United States, 1992-2003. Ann Intern Med 2008;148:728-36.

39 Marur S, D'Souza G, Westra WH, et al. HPV-associated head and neck cancer: a virus-related cancer epidemic. Lancet Oncol 2010:11:781-9.

40 Leemans CR, Braakhuis BJ, Brakenhoff RH. The molecular biology of head and neck cancer. Nat Rev Cancer 2011;11:9-22.

41 Kreimer AR, Clifford GM, Boyle P, et al. Human papillomavirus types in head and neck squamous cell carcinomas worldwide: a systematic review. Cancer Epidemiol Biomarkers Prev 2005;14:467-75.

42 Dutch National Cancer Registry. http://cijfersoverkanker.nl

43 Braakhuis BJ, Visser O, Leemans CR. Oral and oropharyngeal cancer in the Netherlands between 1989 and 2006: increasing incidence, but not in young adults. Oral Oncol 2009;45:e85-9.

44 De Melker H, Kenter G, van Rossum T, et al. Developments in HPV vaccination [in Dutch]. Ned Tijdschr Geneeskd 2012:156:A5410.

45 Bogaards JA, Xiridou M, Coupé VM, et al. Model-based estimation of viral transmissibility and infection-induced resistance from the age-dependent prevalence of infection for 14 high-risk types of human papillomavirus. Am J Epidemiol 2010;171:817-25.

46 Bogaards JA, Coupé VM, Xiridou M, et al. Long-term impact of human papillomavirus vaccination on infection rates, cervical abnormalities, and cancer incidence. Epidemiology 2011;22:505-15.

47 Rothman KJ, Greenland S. Modern epidemiology. 2nd ed. LippincottRaven, 1998

48 Bakker F, Vanwesenbeeck I., eds. Sexual health in the Netherlands in 2006 [in Dutch]. Eburon Academic Publishers, 2006.

49 Lont AP, Kroon BK, Horenblas S, et al. Presence of high-risk human papillomavirus DNA in penile carcinoma predicts favorable outcome in survival. Int / Cancer 2006:119:1078-81.
50 Heideman DA, Waterboer T, Pawlita M, et al. Human papillomavirus-16 is the predominant type etiologically involved in penile squamous cell carcinoma. J Clin Oncol 2007:25:4550-6.

51 Rietbergen MM, Leemans CR, Bloemena E, et al. Increasing prevalence rates of HPV attributable oropharyngeal squamous cell carcinomas in the Netherlands as assessed by a validated test algorithm. Int J Cancer 2013;132:1565-71

52 Miralles-Guri C, Bruni L, Cubilla AL, et al. Human papillomavirus prevalence and type distribution in penile carcinoma. J Clin Pathol 2009:62:870-8

53 Hoots BE, Palefsky JM, Pimenta JM, et al. Human papillomavirus type distribution in anal cancer and anal intraepithelial lesions. Int / Cancer 2009; $124: 2375-83$

54 Smeets SJ, Hesselink AT, Speel EJ, et al. A novel algorithm for reliable detection of human papillomavirus in paraffin embedded head and neck cancer specimen. Int / Cancer 2007;121:2465-72.

55 O’Rorke MA, Ellison MV, Murray LJ, et al. Human papillomavirus related head and neck cancer survival: a systematic review and meta-analysis. Oral Oncol 2012;48:1191-201.

56 Conway EL, Farmer KC, Lynch WJ, et al. Quality of life valuations of HPV-associated cancer health states by the general population. Sex Transm Infect 2012;88:517-21.

57 Ault KA., Future II Study Group. Effect of prophylactic human papillomavirus L1 virus-like-particle vaccine on risk of cervical intraepithelial neoplasia grade 2 , grade 3 , and adenocarcinoma in situ: a combined analysis of four randomised clinical trials. Lancet 2007;369:1861-8

58 Paavonen J, Naud P, Salmerón J, et al. Efficacy of human papillomavirus (HPV)-16/18 AS04-adjuvanted vaccine against cervical infection and precancer caused by oncogenic HPV types (PATRICIA): final analysis of a double-blind, randomised study in young women. Lancet 2009;374:301-14

59 Reuschenbach M, Kansy K, Garbe K, et al. Lack of evidence of human papillomavirus-induced squamous cell carcinomas of the oral cavity in Southern Germany. Oral Oncol 2013;49:937-42.

60 Sanders AE, Slade GD, Patton LL. National prevalence of oral HPV infection and related risk factors in the US adult population. Oral Dis 2012;18:430-41.

61 Lingen MW, Xiao W, Schmitt A, et al. Low etiologic fraction for high-risk human papillomavirus in oral cavity squamous cell carcinomas. Oral Oncol 2013;49:1-8

62 Näsman A, Attner P, Hammarstedt L, et al. Incidence of human papillomavirus (HPV) positive tonsillar carcinoma in Stockholm, Sweden: an epidemic of viral-induced carcinoma? Int / Cancer 2009:125:362-6.

63 Chaturvedi AK, Engels EA, Pfeiffer RM, et al. Human papillomavirus and rising oropharyngeal cancer incidence in the United States. J Clin Oncol 2011;29:4294-301.

64 Burger EA, Sy S, Nygard M, et al. Prevention of HPV-related cancers in Norway: cost-effectiveness of expanding the HPV vaccination program to include pre-adolescent boys. PLoS One 2014;9:e89974.

65 Laprise JF, Drolet M, Boily MC, et al. Comparing the cost-effectiveness of two- and three-dose schedules of human papillomavirus vaccination: a transmission-dynamic modelling study. Vaccine 2014;32:5845-53.

66 Vogt SL, Gravitt PE, Martinson NA, et al. Concordant oral-genital HPV infection in South Africa couples: evidence for transmission. Front Oncol 2013;3:303.

67 Paavonen J, Jenkins D, Bosch EX et al. Efficacy of a prophylactic adjuvanted bivalent $\mathrm{L} 1$ virus-like-particle vaccine against infection with human papillomavirus types 16 and 18 in young women: an interim analysis of a phase III double-blind, randomised controlled trial. Lancet 2007:369:2161-70.

68 Herrero R, Quint W, Hildesheim A, et al. Reduced prevalence of oral human papillomavirus (HPV) 4 years after bivalent HPV vaccination in a randomized clinical trial in Costa Rica. PLoS One 2013; e68329.

69 Coupé VM, van Ginkel J, de Melker HE, et al. HPV16/18 vaccination to prevent cervical cancer in The Netherlands: model-based costeffectiveness. Int J Cancer 2009;124:970-8.

70 McDonald SA, van Lier A, Plass D, et al. The impact of demographic change on the estimated future burden of infectious diseases: examples from hepatitis B and seasonal influenza in the Netherlands. BMC Public Health 2012:12:1046.

71 Van Rijckevorsel G, Whelan J, Kretzschmar M, et al. Targeted vaccination programme successful in reducing acute hepatitis $B$ in men having sex with men in Amsterdam, the Netherlands. J Hepatol 2013;59:1177-83.

72 Schim van der Loeff MF, Mooij SH, Richel O, et al. HPV and anal cancer in HIV-infected individuals: a review. Curr HIV/AIDS Rep 2014:11:250-62.

(c) BMJ Publishing Group Ltd 2015

Appendix 1: Computational framework

Appendix 2: Estimation of parameters

Appendix 3: Supplementary figures A-B 